\title{
Inorganic caesium lead iodide perovskite solar cells
}

Giles E. Eperon ${ }^{\mathrm{a}}$, Giuseppe M. Paternò ${ }^{\mathrm{bc}}$, Rebecca J. Sutton ${ }^{\mathrm{a}}$, Andrea Zampetti ${ }^{\mathrm{bc}}$, Amir Abbas Haghighirad ${ }^{\mathrm{a}}$, Franco Cacialli ${ }^{\text {bc }}$ and Henry J. Snaith ${ }^{\mathrm{a} *}$

${ }^{a}$ Department of Physics, Clarendon Laboratory, University of Oxford, Oxford OX1 3PU, United Kingdom

${ }^{b}$ Department of Physics and Astronomy, University College London, Gower Street, London WC1E 6BT, UK ${ }^{c}$ London Centre for Nanotechnology, University College London, Gordon Street, London WC1E 6BT, UK

*Email: h.snaith1@physics.ox.ac.uk

\begin{abstract}
The vast majority of perovskite solar cell research has focused on organic-inorganic lead trihalide perovskites. Herein, we present working inorganic $\mathrm{CsPbl}_{3}$ perovskite solar cells for the first time. $\mathrm{CsPbl}_{3}$ normally resides in a yellow non-perovskite phase at room temperature, but by careful processing control and development of a low-temperature phase transition route we have stabilised the material in the black perovskite phase at room temperature. As such, we have fabricated solar cell devices in a variety of architectures, with current-voltage curve measured efficiency up to $2.9 \%$ for a planar heterojunction architecture, and stabilised power conversion efficiency of $1.7 \%$. The well-functioning planar junction devices demonstrate long-range electron and hole transport in this material. Importantly, this work identifies that the organic cation is not essential, but simply a convenience for forming lead triiodide perovskites with good photovoltaic properties. We additionally observe significant rate-dependent currentvoltage hysteresis in $\mathrm{CsPbl}_{3}$ devices, despite the absence of the organic polar molecule previously thought to be a candidate for inducing hysteresis via ferroelectric polarisation. Due to its space group and lack of a polar component, $\mathrm{CsPbl}_{3}$ cannot be a ferroelectric material, and as such we can conclude that ferroelectricity is not required to explain current-voltage hysteresis in perovskite solar cells. Our report of working inorganic perovskite solar cells paves the way for further developments likely to lead to much more thermally stable perovskite solar cells and other optoelectronic devices.
\end{abstract}




\section{Introduction}

The meteoric rise of hybrid organic-inorganic perovskite solar cells has seen power conversion efficiencies rise from $3.8 \%$ to over $20 \%$ in merely a couple of years, with more than 1000 scientific publications on the topic. ${ }^{1-6}$ The most studied materials are methylammonium lead triiodide $\left(\mathrm{CH}_{3} \mathrm{NH}_{3} \mathrm{Pbl}_{3}\right.$ or $\left.\mathrm{MAPbl}\right)$ and formamidinium lead triiodide $\left.\left(\mathrm{NH}_{2} \mathrm{CH}_{2} \mathrm{NH}_{3} \mathrm{Pbl}_{3} \text { or } \mathrm{FAPbl}\right)_{3}\right)$, which are 3-dimensional hybrid organic-inorganic perovskite semiconductors with the generic chemical formula $A B X_{3}{ }^{7,8}$ These materials exhibit long-range electron and hole diffusion lengths, low exciton binding energies, high carrier mobilities and suitable bandgaps for making solar cells and other optoelectronic devices. ${ }^{7,9-12}$ Whilst the organic cation enables stabilised $\mathrm{ABX}_{3}$ perovskite which could not be formed by simply employing elements (ions) within the periodic table, one concern of these hybrid perovskites, when compared to conventional thin film compound semiconductors, is the inclusion of the organic cation: The hybrid perovskites have considerably lower thermal decomposition temperatures $\left(\sim 300^{\circ} \mathrm{C}\right.$ in bulk and between 150 to $200{ }^{\circ} \mathrm{C}$ in thin film) than conventional inorganic semiconductors. A poignant question to ask is whether the organic cation is an essential component in high efficiency metal halide perovskite solar cells, or can all inorganic metal halide perovskite solar cells be developed to match the hybrid materials on efficiency, and simultaneously match conventional PV materials on fundamental thermal stability? In addition, whilst these hybrid perovskites form high quality thin film semiconductors and subsequently high efficiency solar cells, in certain configurations they exhibit a current-voltage hysteresis on the timescale of seconds, inhibiting the maximum performance being achieved.$^{13-15}$ Recently there has been much speculation about the origin of this hysteresis, with the two main suggestions being 1 ) that it is due to the motion of charged defects in solar cells under operating conditions, leading to beneficial or detrimental bias-dependent charge extraction efficiency, ${ }^{16-19}$ or 2 ) that it is due to a ferroelectric polarisation, originating from aligned dipolar organic molecules in the lattice. ${ }^{20-24}$ There is evidence for both theories and thus far no studies have conclusively ruled out either possibility. Replacement of the polar organic molecule with a non-polar component would be an ideal test for the ferroelectric theory, since ferroelectricity of the $\mathrm{MAPbl}_{3}$ perovskite is thought to arise from alignment of the MA molecular dipoles throughout the lattice. If the polar organic molecule is responsible for the hysteretic effect, replacing it with a non-polar component should result in hysteresis-free devices. There have been reports of all inorganic caesium tin iodide perovskite solar cells, which do replace the organic component and also the lead cation. ${ }^{25,26}$ However, the efficiencies are very low and these are fabricated on mesoporous titania, a structure that is known to mitigate hysteretic effects. ${ }^{13}$ Additionally, fabrication of Sn-based devices remains challenging due to susceptibility for the $\mathrm{Sn}^{2+}$ ion to oxidize to $\mathrm{Sn}^{4+} \cdot{ }^{27}$ Caesium lead iodide, the perovskite formed by substituting the organic cation in $\mathrm{MAPbl}_{3}$ with caesium, has not yet been reported in functioning solar cells, likely due to the fact that the desired black ( $\sim 1.73 \mathrm{eV}$ bandgap) cubic perovskite phase is not stable at room temperature at ambient conditions, making fabrication challenging. ${ }^{8}$ The material generally transforms to the yellow $\mathrm{NH}_{3} \mathrm{CdCl}_{3}$ non-perovskite structure at room temperature. ${ }^{28}$ Solar cells with this yellow phase have been reported, but did not function at more than $0.09 \%$ PCE. ${ }^{29}$ Herein, by processing the material in a totally air-free environment, allowing us to stabilise it in its black phase, we fabricate working black phase caesium lead iodide solar cells for the first time, and show that such fully inorganic devices do display significant hysteresis in the current-voltage measurement. As such, we can comprehensively conclude that rotational alignment of the organic polar molecule in hybrid perovskite solar cells is not necessary for inducing current-voltage hysteresis. Moreover, the respectable starting performance of these inorganic perovskite devices shows that the hybrid nature of the previously explored perovskites is not critical to fabrication of working solar cells. The solar cells function even in a thin-film planar heterojunction architecture, demonstrating that this material has good ambipolar transport properties and a significant diffusion length. 


\section{Results and discussion}

Caesium lead iodide $\left(\mathrm{CsPb}_{3}\right)$ has been previously synthesised as single crystals and as nanocrystals, and as a dopant in methylammonium lead iodide films in solar cells. ${ }^{8,29-33}$ It can be solution-processed in a similar manner to the hybrid lead halide perovskites; upon formation at room temperature it exhibits a yellow orthorhombic phase with wide bandgap, unsuitable for solar cell applications. ${ }^{8}$ Upon heating, it can form a black cubic perovskite phase with an optical bandgap of $\sim 1.73 \mathrm{eV} .^{33}$ The phase transition has been reported to occur at temperatures of $\sim 310^{\circ} \mathrm{C}$. ${ }^{8,32}$ However, upon cooling, this phase is unstable in ambient conditions at room temperature, returning to the yellow non-perovskite phase in a matter of minutes. Practically, we found that when spin-coating a thin film of material, heating at temperatures of $\geq 335^{\circ} \mathrm{C}$ was necessary to form the black phase, and that after returning to the yellow phase when exposed to ambient conditions, reheating would return it to the black phase. Notably, we found that when the film was never exposed to ambient air, it remained 'frozen' in the black phase even at room temperature, for a matter of weeks at least. Thus, by processing full devices in completely air-free systems, we were able to fabricate thin films and full solar cell devices. We were able to form smooth and uniform thin films of black phase $\mathrm{CsPbl}_{3}$ by spin-coating a 1:1 Csl: $\mathrm{Pbl}_{2}$ solution in $\mathrm{N}, \mathrm{N}$-dimethylformamide (DMF) and heating to $335^{\circ} \mathrm{C}$. However, $335^{\circ} \mathrm{C}$ is still a relatively high temperature, rendering the conversion impractical for a number of device architectures and substrates, such as temperature sensitive c-Si solar cells and hence relevant for tandem cell applications. We found that by adding a small amount of hydroiodic acid to the precursor solution prior to spin-coating, an additive commonly employed to enhance the solubility of perovskite precursors allowing uniform film formation, ${ }^{7,34}$ we were able to convert from the yellow to the black phase at only $100^{\circ} \mathrm{C}$. Using this additive route, we were able to form uniform and smooth thin films of black $\mathrm{CsPbl}_{3}$ by spin-coating the precursor solution plus additive and annealing at only $100^{\circ} \mathrm{C}$ for 10 minutes. 


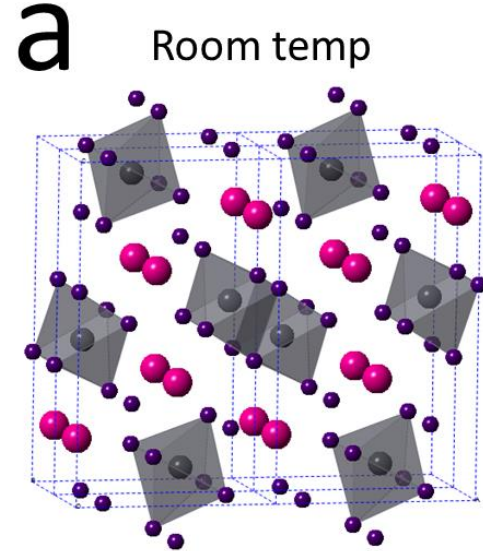

Orthorhombic Pnma Yellow phase

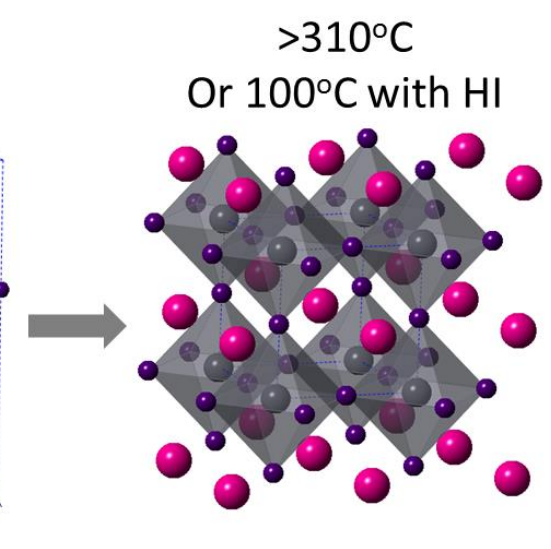

Cubic

Pm-3m

Black phase
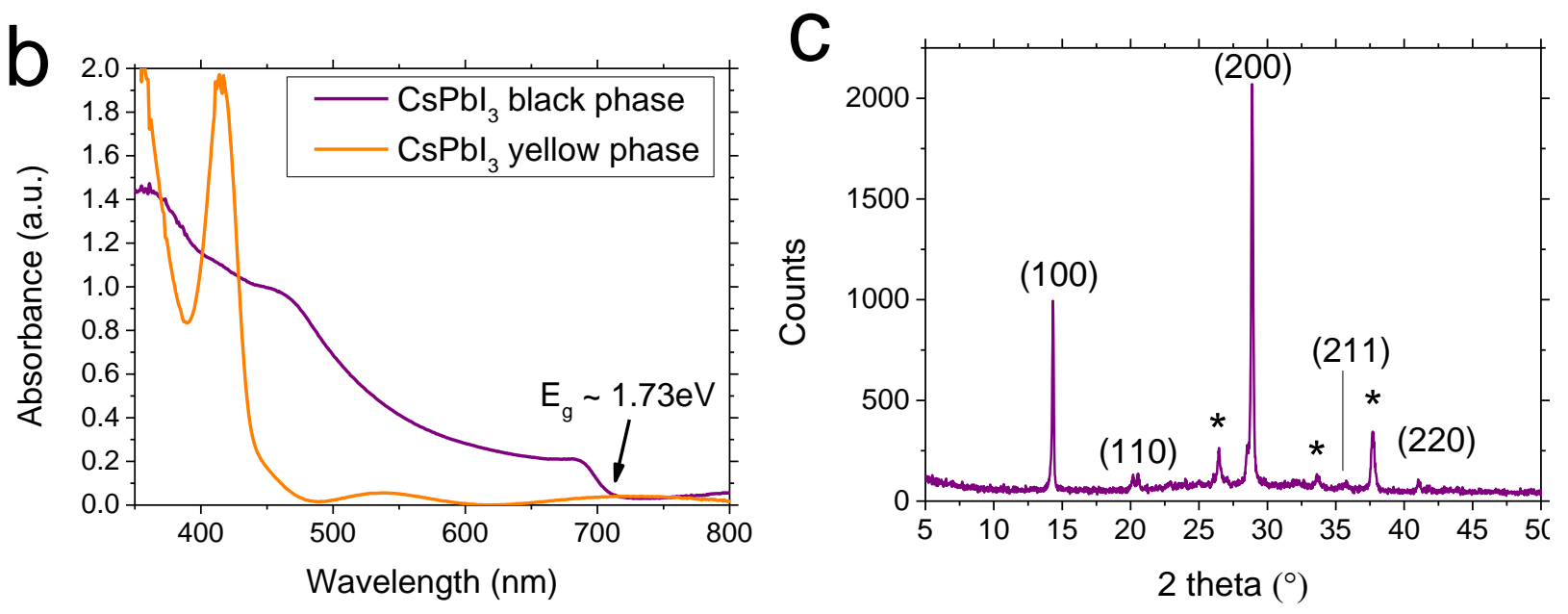

Fig 1. Material properties of $\mathrm{CsPbl}_{3}$ a) Diagrammatic structure of $\mathrm{CSPbl}_{3}$ phases ${ }^{8,30}$. b) Absorbance spectra of black and yellow phases of $\mathrm{CsPbl}_{3}$ thin films. c) X-ray diffraction spectra (XRD) of $\mathrm{CsPbl}_{3}$ thin film in black phase, with peaks assigned to a cubic $(P m-3 m)$ lattice with $a=6.1769(3) A$. Peaks marked with * are those assigned to the FTO substrate. The XRD was performed in air, with the perovskite film coated with polymethylmethacrylate (PMMA) to minimise exposure to air and inhibit the transformation into the yellow phase.

In Fig. 1 we show the material properties of $\mathrm{CsPbl}_{3}$ yellow and black phase thin films. The crystal structures of the yellow and black phases are shown in Fig. 1a). Absorbance spectra (Fig. 1b) agree with previous observations and indicate a material with bandgap of $\sim 1.73 \mathrm{eV}$ for the black phase, and a material absorbing only below $\sim 440 \mathrm{~nm}(2.82 \mathrm{eV})$ in the yellow phase. Refinement of $\mathrm{x}$-ray diffraction data for the black phase (Fig. 1c) indicates a cubic perovskite structure with lattice constant $a=6.1769(3) \AA$ and space group Pm-3m (no. 221). ${ }^{8,30}$ We note that this is not a ferroelectric space group, so this material cannot sustain ferroelectricity in the classical manner, by distortion of the lattice.

The addition of $\mathrm{HI}$ did not result in any obvious changes to the optical properties of the material, as we show in Fig. 2a; the absorption spectrum is essentially identical for the low and high temperature processed material. However, we noticed that in addition to forming the black phase at lower temperature, the films processed with $\mathrm{HI}$ at low temperature were stable in the black phase for significantly longer when exposed 
to air than the high-temperature processed films - hours rather than minutes. This implies that the films processed with $\mathrm{HI}$ are producing a material with a more energetically favourable black phase - it requires less energy input to form it and it is more stable once formed. Considering the role of $\mathrm{HI}$, and the mechanism by which it allows us to form the black phase at a lower temperature, we carried out a more indepth characterisation of the material formed with and without $\mathrm{HI}$. 

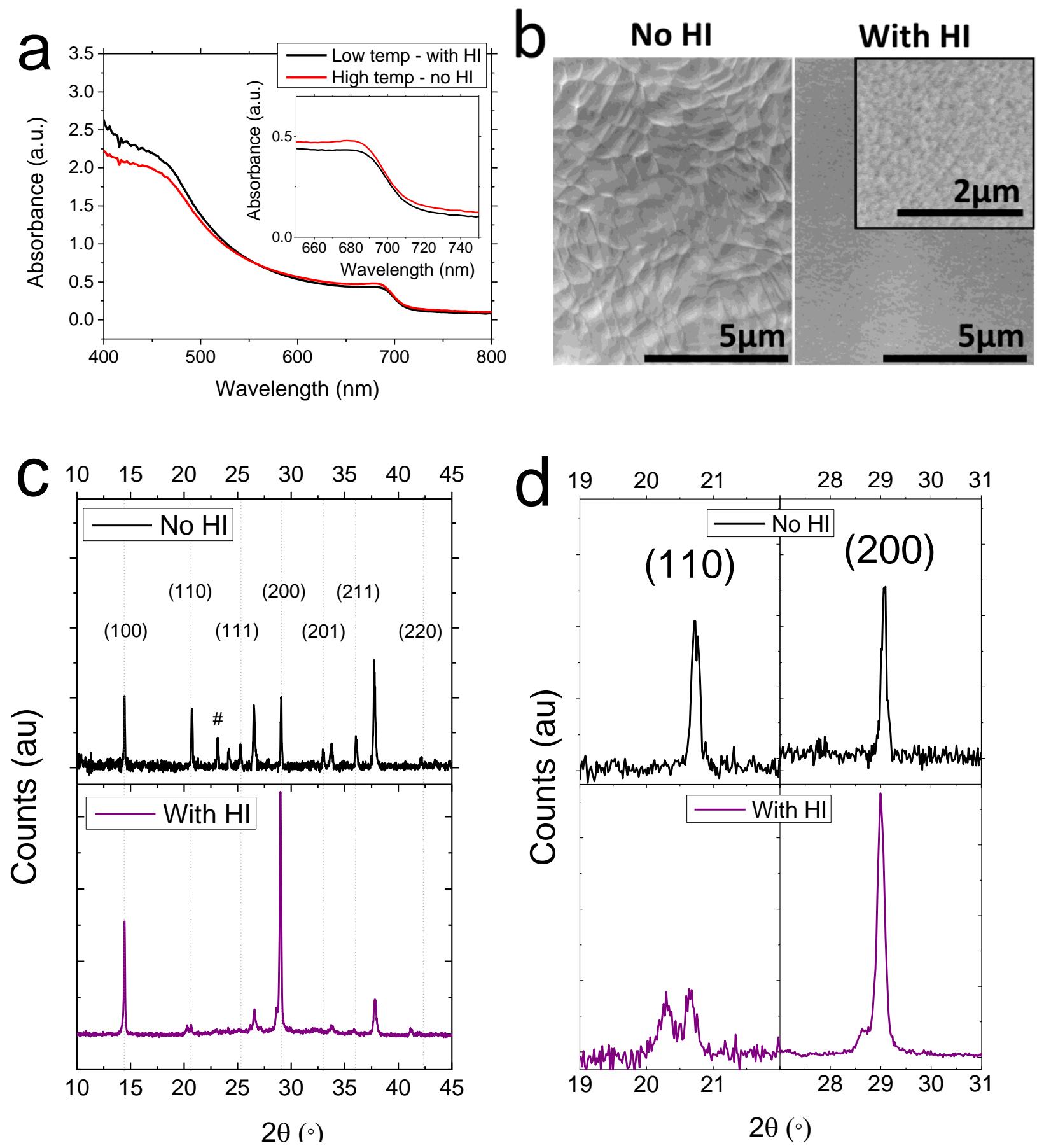

Fig. 2. a) Comparison of absorbance spectra of films fabricated at low and high temperatures (with and without the hydroiodic acid additive) on $\mathrm{FTO} / \mathrm{compact} \mathrm{TiO}_{2}$ substrate, which is representative of the morphology on all substrates. Inset: magnification of onset. b)Scanning electron micrographs of films fabricated without and witt $\mathrm{HI}$ additive, annealed at high and low temperature respectively. Inset: magnification of film fabricated with HI showing small grain size. c) Comparison of XRD spectra of films processed with and without HI. Assigned peaks are marked; peaks labelled with a \# are assigned to some yellow phase present due to degradation in the film without $\mathrm{HI}$ (full spectrum of yellow phase in SI). d) Magnification of the (110) and (200) peaks to show peak splitting and shoulder in film processed with $\mathrm{HI}$.

As we show in Fig. 2b, scanning electron microscope characterisation of the surface of black phase films formed with and without $\mathrm{HI}$ (annealed at low and high temperature respectively), indicate a significant difference. Both films appear very uniform and smooth, but the grain size of the films formed without $\mathrm{HI}$ is 
very large, whereas with $\mathrm{HI}$, the grains are significantly smaller - only on the scale of $~ 100 \mathrm{~nm}$, compared to almost microns in the film processed without HI. Comparing the X-ray diffraction spectra (Fig. $2 \mathrm{c}$ and $2 \mathrm{~d}$ ), we notice that although the overall majority crystal structure appears identical in the two films, there is a different degree of orientation (comparing the magnitude of the (100) and (200) peaks with the other peaks observed) - the film processed with $\mathrm{HI}$ has a more pronounced orientation. Moreover, looking closely at the (110) and (200) peaks (Fig 2d), a further difference becomes evident. The (110) peak is in fact split in the film processed with $\mathrm{HI}$, appearing as a single peak only in the film processed without HI. This second peak cannot be assigned to any possible impurity, nor degradation to the yellow phase (spectrum in SI). The (200) peak exhibits a small shoulder in the HI processed film, and is a clear single peak in the film without HI. Peak splitting such as this is often related to the presence of strain in a crystal; as such we propose that the film processed with $\mathrm{HI}$ has a slightly strained crystal lattice. ${ }^{35}$ This strain could then be responsible for allowing the lower temperature phase transition; strain has previously been observed to induce crystal phase transitions, and serves to completely shift the phase diagram for a material. ${ }^{36-39}$ The role of $\mathrm{HI}$ in creating this lattice strain is likely related to formation of the smaller grains, causing the strain in the lattice. The small crystals presumably result from faster crystallisation from the solution containing $\mathrm{HI}$, which could be due to the $\mathrm{HI}$ being driven off more rapidly than pure DMF, or reduced solubility of the Cs precursor in a solution containing HI. We note that this would be opposite to the behaviour normally observed for $\mathrm{MAPbl}_{3}$ or $\mathrm{FAPbl}_{3}$, but given the replacement of the organic component with Cs would not be unprecedented. In either case though, HI clearly induces the formation of smaller grains, and this is likely responsible for stabilising the black phase at lower temperature. We note that in the previous report of $\mathrm{CsPbl}_{3}$ nanocrystals, Protesescu et al observed that the smaller the nanocrystals, the more stable they were in the black phase, with the smallest nanocrystals being stable in the black phase for months. ${ }^{30}$ This fits well with our observations and reinforces our hypothesis, suggesting that the grain size is of critical importance for stabilisation of the black phase at low temperature. As an aside, this points towards controlling grain boundaries and surface states being of critical importance for these inorganic perovskites, if small grains are a prerequisite of stable crystal phase.

Having ascertained that we have indeed formed $\mathrm{CsPbl}_{3}$ stably in the black cubic phase, and that we can maintain it in this phase for a long period of time by processing in air-free environments, we fabricated solar cell devices. We made solar cells in both the planar heterojunction and infiltrated mesoporous $\mathrm{TiO}_{2}$ architectures, as planar heterojunctions will function only if the material is such that photoexcitation generates free carriers which are able to reach the opposite sides of the device before recombining. Infiltrating the perovskite into mesoporous titania allows materials with worse transport properties to function effectively, with the material acting as a "sensitizer", and transferring photoexcited carriers rapidly into the mesoporous titania and hole transporting layer as appropriate. Additionally, the low temperature processing route allowed us to fabricate 'inverted' planar heterojunction devices, based on a Poly(3,4ethylene dioxythiophene) doped with poly(styrene sulfonate) (PEDOT:PSS) coated substrate with the $\mathrm{n}$ type phenyl-C61-butyric-acid-methyl ester (PCBM) collection layer on top. We show diagrammatic representations of the different architectures employed in Fig. 2a. 

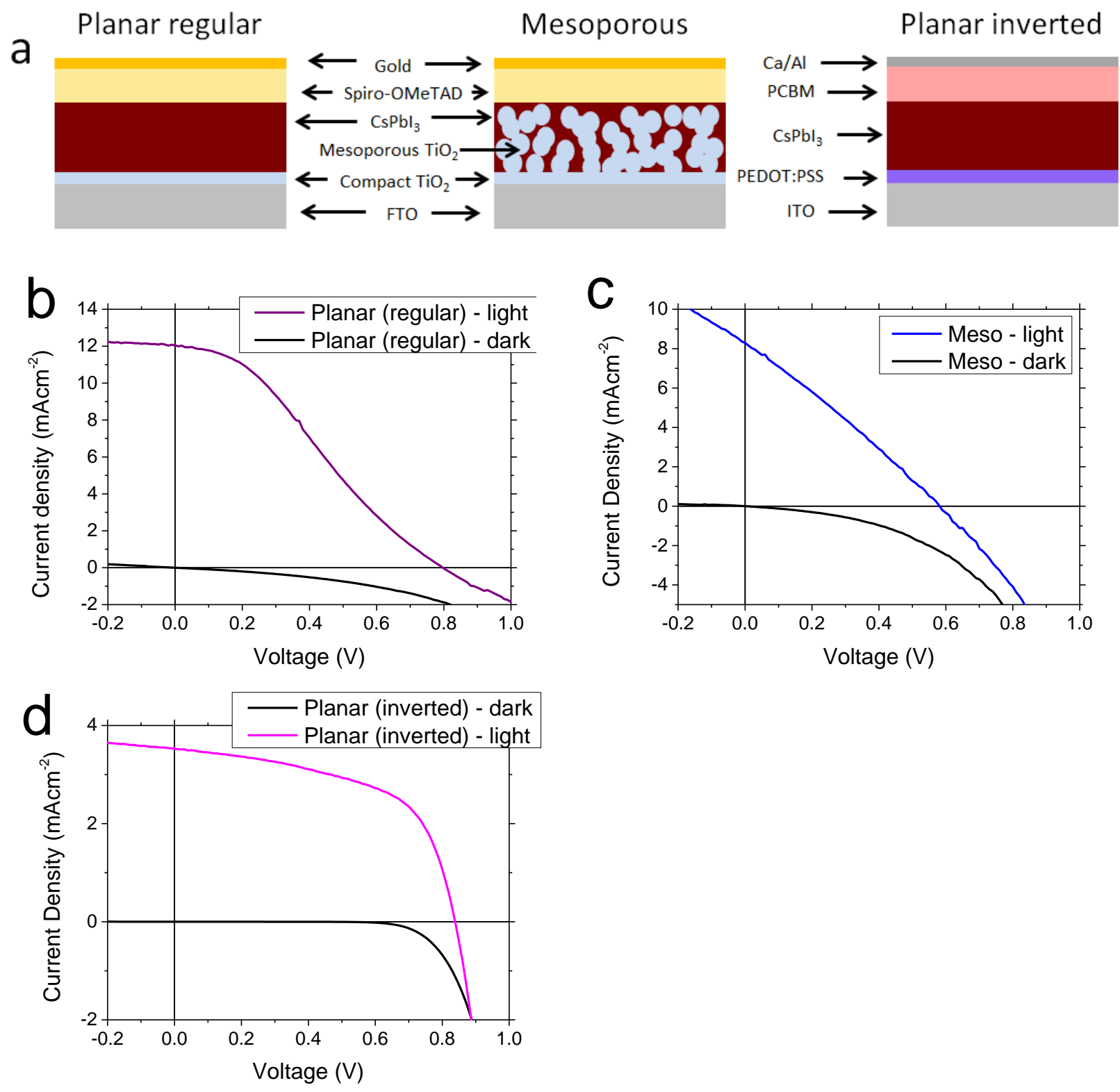

Fig 2. Solar cell properties. a) Schematic of the architectures used. Current-voltage characteristics measured under simulated AM1.5G illumination, scanning from forward to reverse bias at $0.1 \mathrm{~V} / \mathrm{s}$, for regular planar heterojunctions (b), mesoporous titania based devices (c), and inverted planar heterojunctions (d). Devices were unencapsulated and were measured in vacuum conditions.

The perovskite, optionally infiltrated within a $400 \mathrm{~nm}$ thick layer of mesoporous $\mathrm{TiO}_{2}$, is sandwiched between electron-selective and hole-selective contacts of compact $\mathrm{TiO}_{2}$ and Spiro-OMeTAD respectively for the 'regular' $n$-i-p devices and PCBM and PEDOT:PSS for the 'inverted' $p$-i-n devices. When not infiltrated, the perovskite has thickness of $\sim 220 \mathrm{~nm}$. The Spiro-OMeTAD is doped with Spiro(TFSI) ${ }_{2}$ and tertbutylpyridine; the Spiro(TFSI) 2 negates the normal requirement for doping via air exposure. ${ }^{40}$ We show the current-voltage (JV) characteristics, measured under AM1.5 illumination and scanning from forward to reverse bias, in Fig. $2 \mathrm{~b}, 2 \mathrm{c}$ and $2 \mathrm{~d}$. We observe that the regular architecture planar perovskite solar cells generate up to $12 \mathrm{mAcm}^{-2}$ short-circuit current density, and an open-circuit voltage of $\sim 0.8 \mathrm{~V}$. This results in 2.9\% power conversion efficiency (PCE) for this fast JV scan. The mesoporous titania devices however do not perform as effectively, generating lower open-circuit voltage, fill factor, and short-circuit current. This results in only $1.3 \%$ PCE. The planar inverted devices generate a lower current, but a good fill factor and 
open-circuit voltage, resulting in $1.7 \%$ PCE. We note that we also fabricated high-temperature processed $\mathrm{CsPb}_{3}$ devices in the regular planar and mesoporous structures; these devices behaved very similarly to the low-temperature processed films. The planar regular devices and the mesoporous devices are clearly subject to imperfect shunt and series resistances, and possibly non-optimal interface contacts causing the $\mathrm{s}$-shape of the planar device. Clearly, they are not optimised devices, but they do function. Further work will be necessary to understand the limitations of these initial devices, and fabricate high-efficiency solar cells. These will likely be enabled by optimising deposition techniques and annealing protocols, as has been responsible for the current high efficiencies of the more studied $\mathrm{MAPbl}_{3}$ and $\mathrm{FAPbl}_{3}$ devices. ${ }^{34,41,42}$

The fact that the planar devices function is integral to the understanding of this material. A functioning planar device means that because charges are extracted and a significant short-circuit current generated, carriers must be able to travel across the film before recombining. This implies that both electrons and holes must have a significant diffusion length in caesium lead triiodide. Moreover, it implies that the exciton binding energy is low enough that most excited carriers in the film are present as free carriers as opposed to excitons. The fact that the planar devices outperform the mesoporous devices indicates that transport in the perovskite is likely superior to transport in the mesoporous titania, and indicates that there is no need for the mesostructured approach for this all inorganic perovskite.

As previously discussed, the issue of current-voltage hysteresis, exhibiting different JV characteristics at different scan speeds, is a critical issue in the hybrid organic-inorganic perovskite solar cells. Typically regular $n$-i-p structured planar devices display most hysteresis, with mesoporous titania-based devices and inverted devices mitigating the effects of hysteresis. The extent of hysteresis can be ascertained by measuring steady-state power output of the cell, which at maximum power point gives the real sustainable efficiency of the device. Comparing this to the current-voltage sweeps allows us to quantify to what extent the PCE is artificially inflated in the JV scan due to the hysteretic effect. As such, we measured currentvoltage characteristics for these fully inorganic perovskite solar cells at different rates, and also measured the steady-state power output. 


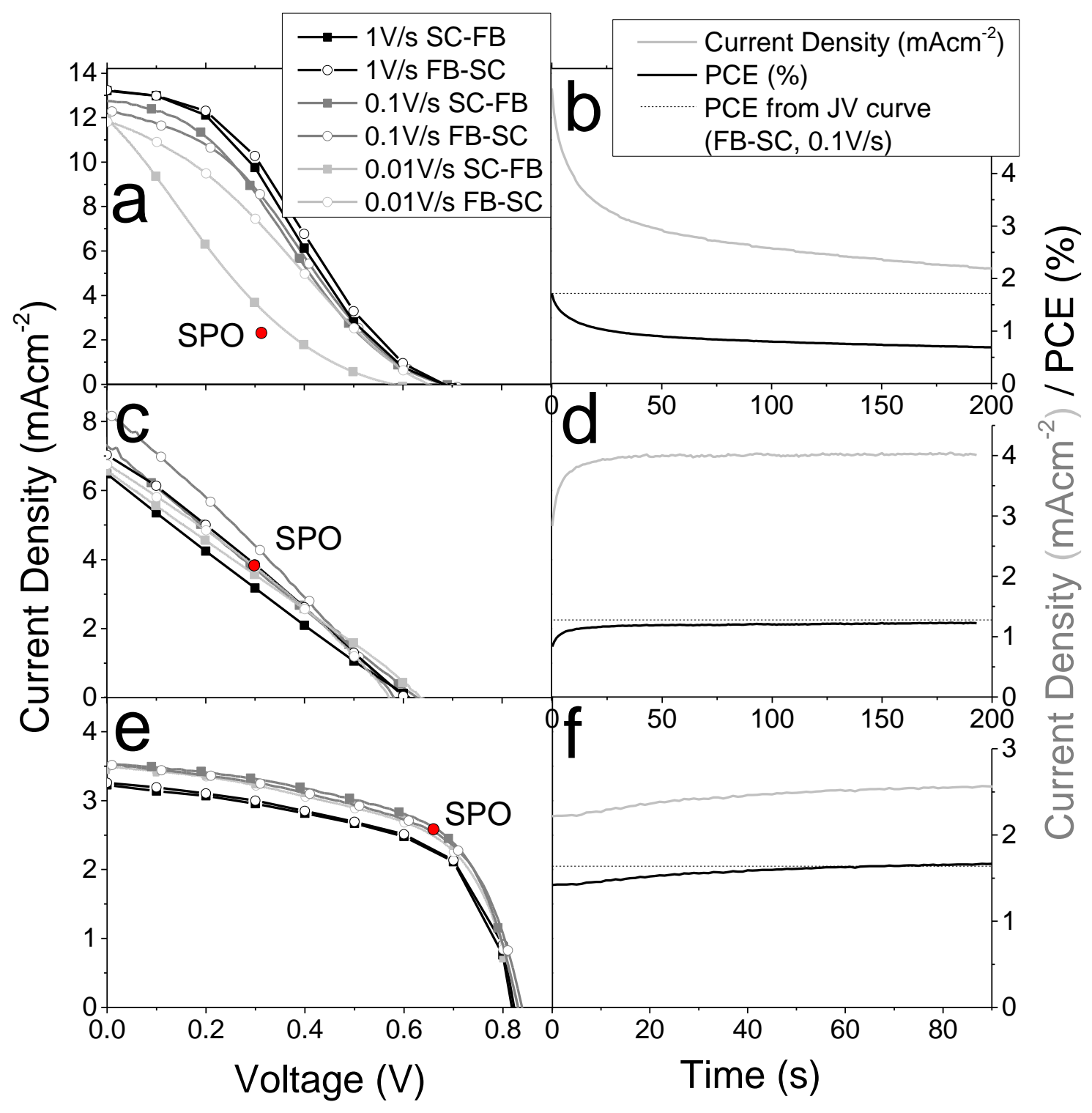

Fig. 3. Hysteresis in inorganic perovskite solar cells. Current-voltage characteristics measured at different sweep rates for a) regular planar devices, c) mesoporous titania devices, and e) inverted planar devices. $F B$ $S C=$ scanning from forward bias to short circuit, SC-FB vice versa.. b), d) and f) show stabilisation of current density and hence PCE measured at the maximum power point determined from FB-SC scan at $0.1 \mathrm{~V} / \mathrm{s}$, compared to the PCE extracted from that JV curve. The final stabilised power output (SPO) is marked on the IV plots as a red circle.

We plot current-voltage characteristics measured at different scan rates on the left, and stabilised power measurements (holding at maximum power point) on the right hand side of Figure 3 . We observe that the regular structure planar device (Fig. 3a) shows large differences in its JV scans depending on scan rate. Faster scans appear to show in particular a superior fill factor (FF), and more similar forward going (SC-FB) and reverse going (FB-SC) scans. At the slower scan speed, there is a very large difference in the forward and reverse scans. Measuring the stabilised power output (Fig. $3 \mathrm{~b}$ ), we show that the stabilised value is significantly below that estimated from the JV scans; indeed, it is lower than any of the scans, no matter the rate or direction. As such, we can conclude that the perovskite device in this architecture does display 
significant hysteresis, with the JV scans overestimating the PCE by a factor of more than 2 . The mesoporous titania devices (Fig. $3 \mathrm{c}$ and d) do also display some variation in hysteresis with scan rate, though not as notable as the planar devices. The stabilised power output rises to a value very similar to the estimated PCE from the JV scan, so we can conclude that while there is some hysteresis in the current-voltage characteristics, it does not critically affect the steady-state response. In the case of the inverted devices, we observe little hysteresis in the JV curves. However, the scans at a very fast rate give a slightly lower shortcircuit current density. The stabilised power output of these devices rises to almost exactly the same as the PCE estimated from the best JV curves, so there appears to be no artificial inflation of the PCE value in this case; hysteresis does not seem to affect these inverted devices detrimentally. We note that there was a similar degree of hysteresis observed in high- and low-temperature processed devices.

From these measurements we can conclude several things. Firstly, current-voltage hysteresis is present in $\mathrm{CsPb}_{3}$ devices. This effect is therefore not unique to the hybrid materials with a dipolar organic molecule. $\mathrm{CsPb}_{3}$ cannot be ferroelectric, either via dipole alignment or by classical lattice distortion, as it does not have a ferroelectric space group. As such, the current-voltage hysteresis displayed here, and likely in other perovskite solar cells, is not resultant from the ferroelectric nature of the material.

Secondly, we observe that the planar regular device shows very significant hysteresis, and over-estimation of the PCE from the JV scans. On the other hand, the mesoporous titania based and inverted planar devices show little overestimation of the PCE, and a much reduced hysteresis in the JV scans. This is in keeping with what has been observed previously for $\mathrm{MAPbl}_{3}$ devices. ${ }^{13,14}$ It is now generally thought that the hysteresis arises due to compensation of an applied bias with an internal built-in field. ${ }^{16,43,44}$ This built-in field acts to reduce recombination after the device has been held at forward bias (i.e. scanning FB-SC); it can allow even devices with poorly selective contacts to function well temporarily by biasing. ${ }^{16}$ This allows carriers to be extracted before recombination, making the solar cell 'better' - this is the reverse scan with the higher efficiency. Devices with contacts that already rapidly extract charge before it recombines will not be so affected by the temporary built-in field, as it is not necessary for efficient extraction of charge. This is likely to be the case for the mesoporous and inverted devices. The large surface area of mesoporous titania allows rapid extraction of electrons, as does the PCBM in the inverted devices. However, the compact titania in the regular planar $n-i-p$ device is not as effective at extracting charge - there is even some evidence for an energy barrier that must be overcome by the temporary built-in field. ${ }^{45}$ As such, it requires the temporary enhancement gained by the device previously being at open-circuit conditions to function well (FB-SC in Fig. 3a). Upon going back to short-circuit, or holding at the maximum power point, the device behaves poorly again (SC-FB in Fig. 3a).

The SPO measurements take 10s of seconds to reach steady state. Furthermore, hysteresis is most severe in JV curves scanned at the slowest rate; the faster scans maintain the device in the beneficial state during scanning. As such, we can conclude that the hysteretic effect takes place on the timescale of seconds.

The main alternative to ferroelectricity proposed as a cause for the hysteretic effect is ion motion within the perovskite film. Charged defect ions could move to compensate an applied field, resulting in the built-in field via charge accumulation at the interfaces or doping of the film at either side. It has been shown that ions can move under bias in perovskite films, and there is mounting evidence that this is also the cause of the current-voltage hysteresis. ${ }^{16,17,44}$ The results presented here agree well with this theory; the timescale of hysteresis observed is as expected for ionic motion.

We note that although the devices presented here are not the most efficient, with further optimisation it is likely that they could perform as well as the hybrid organic-inorganic materials. The fact that we have fabricated working $\mathrm{CsPbl}_{3}$ devices suggests that there is no fundamental property of the hybrid materials that allows them to work as efficient solar cells. We do note however that the maximum open-circuit 
voltage we have generated here under full sun illumination is $0.85 \mathrm{~V}$ and the material has an optical band gap of $1.73 \mathrm{eV}$. Therefore the loss in potential or voltage-deficit, i.e. the difference in energy between the band gap and the open-circuit voltage, is relatively large at $0.88 \mathrm{eV}$. It remains to be seen if we can achieve similarly small voltage deficits as achieved with the organic-inorganic perovskites $(<0.4 \mathrm{eV})$, at which point we would conclusively demonstrate that the inorganic perovskites are as effective PV materials. To motivate such effort, these inorganic materials do not suffer from some limitations of the hybrid materials; notably the thermal stability of $\mathrm{CsPb}_{3}$ is much greater than that of the organic containing materials, where the organic component becomes volatile and easily removed at elevated temperatures. Whilst $\mathrm{MAPbl}_{3}$ is known to degrade even when held at $85^{\circ} \mathrm{C}$ for long periods of time, ${ }^{46}$ rendering it ultimately unsuitable for commercialisation, the black phase of $\mathrm{CsPbl}_{3}$ is stable up to well over $300^{\circ} \mathrm{C}$ - this is clearly a significantly more thermally stable material. If the ambient instability problems of these inorganic materials could be overcome, they could allow long-term efficient operation due to the higher thermal stability. As such, we expect significant further research into inorganic perovskite solar cells to take place, likely to be accompanied by enhanced efficiencies and stability.

\section{Conclusion}

In summary, we have fabricated working inorganic $\mathrm{CsPbl}_{3}$ solar cells for the first time. By carrying out all processing in a totally inert atmosphere, and developing a low temperature phase transition route, we are able to stabilise $\mathrm{CsPbl}_{3}$ films in the black phase at room temperature, and fabricate solar cells in a variety of architectures. This highlights that the organic cation is unlikely to be essential for efficient perovskite solar cells. Despite the fact that $\mathrm{CsPbl}_{3}$ cannot be ferroelectric in any way, we observe significant current-voltage hysteresis in such devices, pointing towards other non-ferroelectric phenomena being the cause of currentvoltage hysteresis. These results pave the way for further optimisation and stabilisation of the inorganic perovskite materials, with potential for even more stable devices than the hybrid organic-inorganic materials currently displaying the highest efficiencies.

\section{Acknowledgements}

This work was in part funded by UCL, EPSRC and the European Commission through the ERC-Stg2011 grant HYPER. GE thanks Oxford Photovoltaics Ltd. and the EPSRC for his CASE studentship award through the Nanotechnology KTN. We also thank the EC Seventh Framework Programme (FP7/2007-2013) under Grant Agreement No. 607585 (OSNIRO). R.S. is a Commonwealth Scholar, funded by the UK government. F.C. is a Royal Society Wolfson Research Merit Award Holder. 


\section{Notes and references}

Electronic Supplementary Information (ESI) available: includes full details of experimental methods, supplementary data and details of calculations used. See DOI: 10.1039/c000000x/

1 A. Kojima, K. Teshima, Y. Shirai and T. Miyasaka, J. Am. Chem. Soc., 2009, 131, 6050-6051.

2 M. M. Lee, J. Teuscher, T. Miyasaka, T. N. Murakami and H. J. Snaith, Science, 2012, 338, 643647.

3 J. Burschka, N. Pellet, S.-J. J. Moon, R. Humphry-Baker, P. Gao, M. K. Nazeeruddin, M. Grätzel and M. Gratzel, Nature, 2013, 499, 316-319.

4 N. J. Jeon, J. H. Noh, W. S. Yang, Y. C. Kim, S. Ryu, J. Seo and S. II Seok, Nature, 2015, 517, 476-480.

5 H. Zhou, Q. Chen, G. Li, S. Luo, T. -b. Song, H.-S. Duan, Z. Hong, J. You, Y. Liu and Y. Yang, Science, 2014, 345, 542-546.

6 NREL, Best Research-Cell Efficiencies, http://www.nrel.gov/ncpv/images/efficiency_chart.jpg, 2015.

7 G. E. Eperon, S. D. Stranks, C. Menelaou, M. B. Johnston, L. M. Herz and H. J. Snaith, Energy Environ. Sci., 2014, 7, 982-988.

8 C. C. Stoumpos, C. D. Malliakas and M. G. Kanatzidis, Inorg. Chem., 2013, 52, 9019-9038.

9 S. D. Stranks, G. E. Eperon, G. Grancini, C. Menelaou, M. J. P. Alcocer, T. Leijtens, L. M. Herz, A. Petrozza and H. J. Snaith, Science, 2013, 342, 341-344.

10 V. D'Innocenzo, G. Grancini, M. J. P. Alcocer, A. R. S. Kandada, S. D. Stranks, M. M. Lee, G. Lanzani, H. J. Snaith and A. Petrozza, Nat. Commun., 2014, 5, 3586.

11 C. Wehrenfennig, G. E. Eperon, M. B. Johnston, H. J. Snaith and L. M. Herz, Adv. Mater., 2014, 26, 1584-1589.

12 T. Leijtens, S. D. Stranks, G. E. Eperon, R. Lindblad, E. M. J. Johansson, J. M. Ball, M. M. Lee, H. J. Snaith, I. J. McPherson, H. Rensmo, J. M. Ball, M. M. Lee and H. J. Snaith, ACS Nano, 2014, 8, 7155-7174.

13 E. L. Unger, E. T. Hoke, C. D. Bailie, W. H. Nguyen, A. R. Bowring, T. Heumuller, M. G. Christoforo and M. D. McGehee, Energy Environ. Sci., 2014, 7, 3690-3698.

14 H. J. Snaith, A. Abate, J. M. Ball, G. E. Eperon, T. Leijtens, N. K. Noel, S. D. Stranks, J. T. Wang, K. Wojciechowski and W. Zhang, J. Phys. Chem. Lett., 2014, 5, 1511-1515.

15 J. a. Christians, J. S. Manser and P. V. Kamat, J. Phys. Chem. Lett., 2015, 6, 852-857.

16 Y. Zhang, M. Liu, G. E. Eperon, T. C. Leijtens, D. McMeekin, M. Saliba, W. Zhang, M. de Bastiani, A. Petrozza, L. M. Herz, M. B. Johnston, H. Lin and H. J. Snaith, Mater. Horiz., 2015, 00, 1-8. 

Mater., 2015, 14, 193-198. Chem. Phys., 2015, 17, 9613-9618. Lett., 2015, 106, 173502.

R. S. Sánchez, V. Gonzalez-pedro, J. Lee, N. Park, Y. S. Kang, I. Mora-sero and J. Bisquert, 2014, $0-4$.

22 Y. Kutes, L. Ye, Y. Zhou, S. Pang, B. D. Huey and N. P. Padture, J. Phys. Chem. Lett., 2014, 5, 3335-3339.

23 S. Liu, F. Zheng, N. Z. Koocher, H. Takenaka, F. Wang and A. M. Rappe, J. Phys. Chem. Lett., 2015, 6, 693-699. Ramesh, M. Asta, M. Graetzel, S. G. Mhaisalkar and N. Mathews, Adv. Mater., 2014, 26, 7122-7127.

27 N. K. Noel, S. D. Stranks, A. Abate, C. Wehrenfennig, S. Guarnera, A. Haghighirad, A. Sadhanala, G. E. Eperon, S. K. Pathak, M. B. Johnston, A. Petrozza, L. Herz and H. Snaith, Energy Environ. Sci., 2014, 7, 3061-3068.

H. Choi, J. Jeong, H.-B. Kim, S. Kim, B. Walker, G.-H. Kim and J. Y. Kim, Nano Energy, 2014, 7, 80-85.

30 L. Protesescu, S. Yakunin, M. I. Bodnarchuk, F. Krieg, R. Caputo, C. H. Hendon, R. X. Yang, A. Walsh and M. V. Kovalenko, Nano Lett., 2015, 150202125655002.

31 O. N. Yunakova, V. K. Miloslavskii and E. N. Kovalenko, Opt. Spectrosc., 2012, 112, 91-96.

32 C. K. Moller, Nature, 1958, 182, 1436.

33 G. E. Eperon, S. D. Stranks, C. Menelaou, M. B. Johnston, L. M. Herz and H. J. Snaith, 2014.

34 A. Manuscript, S. H. Im, J.-H. Heo, H. J. Han, D. Kim and T. Ahn, Energy Environ. Sci., 2015.

35 H. W. Jang, S. H. Baek, D. Ortiz, C. M. Folkman, R. R. Das, Y. H. Chu, P. Shafer, J. X. Zhang, S. Choudhury, V. Vaithyanathan, Y. B. Chen, D. a. Felker, M. D. Biegalski, M. S. Rzchowski, X. Q. Pan, D. G. Schlom, L. Q. Chen, R. Ramesh and C. B. Eom, Phys. Rev. Lett., 2008, 101, 3-6. 
36 A. J. Hatt, N. a. Spaldin and C. Ederer, Phys. Rev. B - Condens. Matter Mater. Phys., 2010, 81, $1-5$.

37 Y. Tokura, Science, 2000, 288, 462-468.

38 F. Tsui, M. C. Smoak, T. K. Nath and C. B. Eom, Appl. Phys. Lett., 2000, 76, 2421-2423.

39 Y. L. Li and L. Q. Chen, Appl. Phys. Lett., 2006, 88, 3-5.

40 W. H. Nguyen, C. D. Bailie, E. L. Unger and M. D. McGehee, J. Am. Chem. Soc., 2014, 136, 10996-11001.

41 N. J. Jeon, J. H. Noh, W. S. Yang and Y. C. Kim, 1-14.

42 W. S. Yang, J. H. Noh, N. J. Jeon, Y. C. Kim, S. Ryu, J. Seo and S. I. Seok, Science, 2015, 1-12.

43 W. Tress, N. Marinova, T. Moehl, S. M. Zakeeruddin, M. K. Nazeeruddin and M. Grätzel, Energy Environ. Sci., 2015.

44 Y. Zhao, C. Liang, H. min Zhang, D. Li, D. Tian, G. Li, X. Jing, W. Zhang, W. Xiao, Q. Liu, F. Zhang and Z. He, Energy Environ. Sci., 2015, 8, 1256-1260.

45 K. Wojciechowski, S. D. Stranks, A. Abate, G. Sadoughi, A. Sadhanala, N. Kopidakis, G. Rumbles, C. Li, R. H. Friend, A. K.-Y. Jen and H. J. Snaith, ACS Nano, 2014, 12701-12709.

46 B. Conings, J. Drijkoningen, N. Gauquelin, A. Babayigit, J. D'Haen, L. D'Olieslaeger, A. Ethirajan, J. Verbeeck, J. Manca, E. Mosconi, F. De Angelis and H. Boyen, Adv. Energy Mater., 2015, 1-8. 


\section{Supplementary information}

\section{Inorganic caesium lead iodide perovskite solar cells}

Giles E. Eperon ${ }^{\mathrm{a}}$, Giuseppe M. Paternò ${ }^{\mathrm{bc}}$, Rebecca J. Sutton ${ }^{\mathrm{a}}$, Andrea Zampetti ${ }^{\mathrm{bc}}$, Amir Abbas Haghighirad ${ }^{\mathrm{a}}$, Franco Caciallibc and Henry J. Snaith ${ }^{\mathrm{a} *}$

${ }^{a}$ Department of Physics, Clarendon Laboratory, University of Oxford, Oxford OX1 3PU, United Kingdom

${ }^{b}$ Department of Physics and Astronomy, University College London, Gower Street, London WC1E 6BT, UK ${ }^{c}$ London Centre for Nanotechnology, University College London, Gordon Street, London WC1E 6BT, UK

*Email: h.snaith1@physics.ox.ac.uk 


\section{Materials and Methods}

Materials. Unless otherwise stated, all materials were purchased from Sigma-Aldrich or Alfa Aesar and used as received. Spiro-OMeTAD was purchased from Borun Chemicals.

Perovskite film fabrication: To form the $\mathrm{CsPbl}_{3}$ precursor solution, $\mathrm{Csl}$ and $\mathrm{Pbl}_{2}$ were dissolved in anhydrous $\mathrm{N}, \mathrm{N}$-dimethylformamide (DMF) in a 1:1 molar ratio, at $0.48 \mathrm{M}$ of each reagent. For the low-temperature processing route, immediately prior to spin-coating, $33 \mu \mathrm{l}$ of hydroiodic acid $(57 \% \mathrm{w} / \mathrm{w})$ was added to $1 \mathrm{ml}$ of the $0.48 \mathrm{M}$ precursor solution to enable low-temperature phase transition. To form films, the precursor was then spin-coated in a nitrogen-filled glovebox at 2000rpm, and annealed at $100^{\circ} \mathrm{C}$ for 10 minutes. For the high-temperature route, no hydroiodic acid was added and films were annealed at $335^{\circ} \mathrm{C}$ for 5 minutes.

Films for optical studies were fabricated on plasma-cleaned microscope glass slides. For solar cells, films were fabricated on fluorine-doped tin oxide (FTO) coated glass (Pilkington, $7 \Omega \square^{-1}$ ) or pre-patterned indium tin oxide (ITO) coated glass (Colorado Concept Coatings LLC, $\sim 20 \Omega \square^{-1}$ ). For the FTO, initially FTO was removed from regions under the anode contact by etching the $\mathrm{FTO}$ with $2 \mathrm{M} \mathrm{HCl}$ and zinc powder. FTO substrates were then cleaned sequentially in hallmanex detergent, acetone, propan-2-ol and oxygen plasma. ITO-coated glass substrates were cleaned in an ultrasonic bath with acetone and propan-2-ol (10 min each step) and treated with oxygen plasma.

Device fabrication: For the regular devices, a $~ 50 \mathrm{~nm}$ hole-blocking layer of compact $\mathrm{TiO}_{2}$ was first deposited on the FTO by spin-coating a mildly acidic solution of titanium isopropoxide in ethanol $(350 \mu \mathrm{l}$ in $5 \mathrm{~mL}$ ethanol with $0.013 \mathrm{M} \mathrm{HCl}$ ) at $2000 \mathrm{rpm}$, and annealed at $500^{\circ} \mathrm{C}$ for 30 minutes. For the mesoporous cells, $400 \mathrm{~nm}$ mesoporous titania was then deposited by spin-coating at 2000rpm a 1:3 dilution of Dyesol 18NR-T paste: ethanol by weight. This was then annealed by ramping slowly to $500^{\circ} \mathrm{C}$ then holding for 30 mins.

Subsequently substrates were transferred to nitrogen atmosphere and from this point, during fabrication and testing, devices were never exposed to ambient air. Perovskite precursor was deposited as described above. The hole-transporting layer was then deposited via spin-coating a solution of $86.9 \mathrm{mg} / \mathrm{ml}$ of $2,2^{\prime}, 7,7^{\prime}$ tetrakis-(N,N-di-p-methoxyphenylamine)9,9'-spirobifluorene (spiro-OMeTAD) in chlorobenzene, with additives of $9.7 \mathrm{mg} / \mathrm{ml}$ Spiro(TFSI) ${ }_{2}$ prepared in-house following a published procedure, ${ }^{1}$ and $10 \mu \mathrm{l} / \mathrm{ml}$ 4-tertbutylpyridine. Spin-coating was carried out in a nitrogen-filled glovebox at 2000rpm. Gold electrodes were then thermally evaporated under a vacuum of $\sim 10^{-6} \mathrm{Torr}$, at a rate of $\sim 0.1 \mathrm{~nm} / \mathrm{s}$, to complete the devices.

For the inverted devices, ITO substrates were used. An electron-blocking layer of PEDOT:PSS (Heraeus Al4083) was deposited by spin-coating at 4000rpm in air and annealing in nitrogen at $140^{\circ} \mathrm{C}$ for 15 mins. The steps following were then carried out inside a nitrogen atmosphere and from this point, during fabrication and testing, devices were never exposed to ambient air. Perovskite precursor was deposited as described above. The electron-extracting layer was deposited by spin-coating a $20 \mathrm{mg} / \mathrm{ml}$ solution of PCBM in chlorobenzene and annealing at $100^{\circ} \mathrm{C}$ for 30 mins. Electrodes for the inverted devices were calcium $(20 \mathrm{~nm})$ followed by aluminium $(100 \mathrm{~nm})$, thermally evaporated under vacuum of $\sim 10^{-6} \mathrm{Torr}$, at a rate of 0.03 $\mathrm{nm} / \mathrm{s}$ and $\sim 0.1 \mathrm{~nm} / \mathrm{s}$ respectively, to complete the devices.

Device characterisation: The current density-voltage $(\mathrm{J}-\mathrm{V})$ curves were measured (2400 Series SourceMeter, Keithley Instruments) under simulated AM $1.5 \mathrm{G}$ sunlight at $100 \mathrm{mWcm}^{-2}$ irradiance generated by an Abet class AAA Sun 3000 simulator, with the intensity calibrated with an Abet Si reference cell. The solar cells were mounted into a vacuum chamber directly from within the glove box, and pumped under vacuum while measuring.

Optical measurements: Absorbance spectra were collected with a Varian Cary 300 UV-Vis spectrophotometer with an internally coupled integrating sphere. 
Materials Characterization: Sample thicknesses were measured using a Veeco Dektak 150 surface profilometer. X-ray diffraction measurements were performed with a Rigaku SmartLab X-ray diffractometer (Rigaku, Tokyo, Japan) using a $\mathrm{K} \alpha$ source wavelength emitted by a $\mathrm{Cu}$ anode $(0.154 \mathrm{~nm})$, using samples coated with a thick layer of polymethylmethacrylate (PMMA) deposited by drop-casting from chlorobenzene. Structural refinements were carried out with GSAS.

\section{X-ray diffraction refinement details}

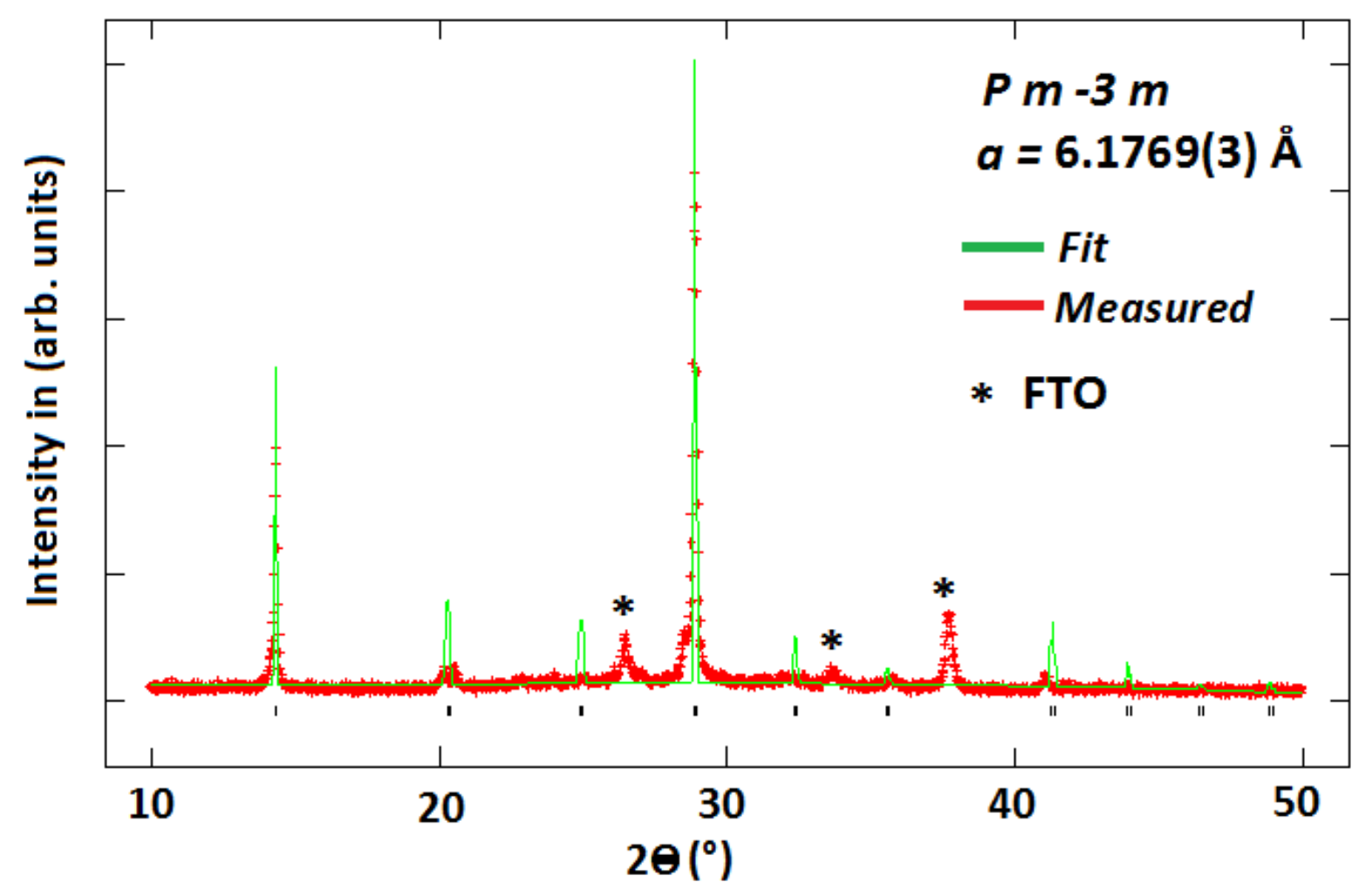

Fig. S1: XRD fitting of $\mathrm{CsPbl}_{3}$ spectrum with Pm-3m space group. Parameters are $a=6.1769 \pm$ $0.0003 \AA$.

\begin{tabular}{|c|c|c|c|c|}
\hline \multirow[t]{2}{*}{ Atom } & \multicolumn{3}{|c|}{ Wyckoff positions } & \multirow[t]{2}{*}{ Site symmetry } \\
\hline & $x$ & $y$ & $z$ & \\
\hline Cs & 0.5 & 0.5 & 0.5 & $m-3 m$ \\
\hline$P b$ & 0 & 0 & 0 & $m-3 m$ \\
\hline I & 0.5 & 0 & 0 & $4 / \mathrm{mmm}$ \\
\hline
\end{tabular}

Density: $5.070 \mathrm{~g} / \mathrm{cm}^{3}$

Volume (unit cell): $235.68 \AA^{3}$

Table ST1: Atomic positons of CSPb/3 in cubic black phase. 


\section{Yellow phase X-ray diffraction pattern}

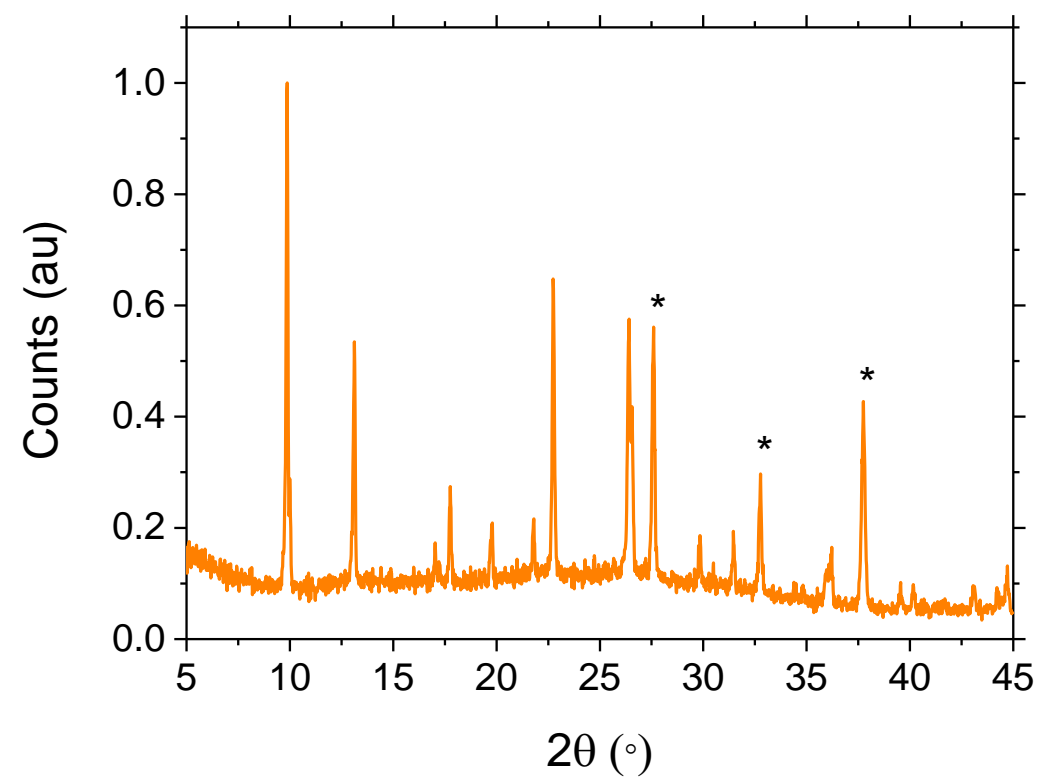

Fig. S2: X-ray diffraction pattern of yellow phase. Peaks marks with $a^{*}$ assigned to the FTO substrate.

\section{References}

1. Nguyen, W. H.; Bailie, C. D.; Unger, E. L.; McGehee, M. D. "Enhancing the Hole-Conductivity of Spiro-OMeTAD without Oxygen or Lithium Salts by Using spiro(TFSI)2 in Perovskite and DyeSensitized Solar Cells". J. Am. Chem. Soc. 2014, 136, 10996-11001. 